\title{
Corrigendum
}

\section{Selective induction of cell cycle arrest and apoptosis in human prostate cancer cells through adenoviral transfer of the melanoma differentiation-associated-7 (mda-7)/interleukin-24 (IL-24) gene}

Yuji Saito, Ryo Miyahara, Began Gopalan, Anya Litvak, Satoshi Inoue, Manish Shanker, Cynthia D Branch, Abner M Mhashilkar, Jack A Roth, Sunil Chada, and Rajagopal Ramesh

Cancer Gene Therapy (2005) 12, 340. doi:10.1038/sj.cgt.7700811

Correction to: Cancer Gene Therapy (2005) 12, 238-247. doi:10.1038/sj.cgt.7700780

Figure 2 was published incorrectly in the above article.

The correct Figure 2 is shown below.

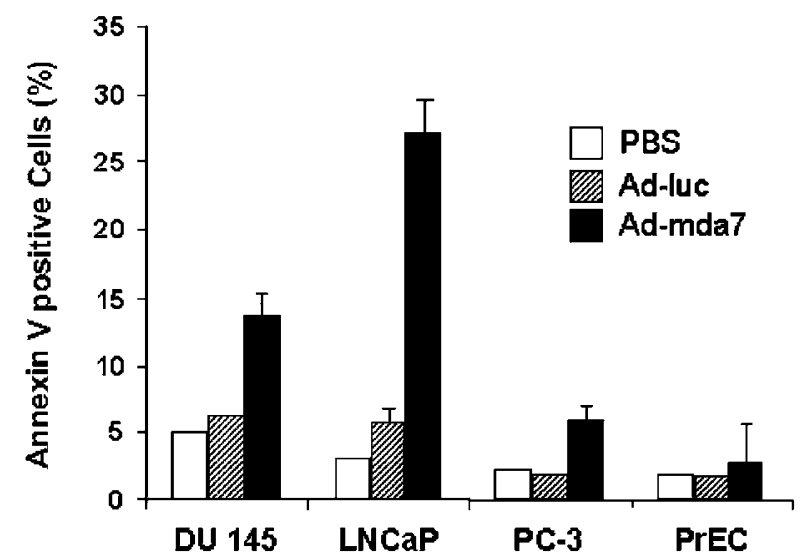

Figure 2 mda-7 expression induces apoptosis in tumor cells but not in normal cells. Tumor cells (DU 145, LNCaP, and PC-3) and normal epithelial cells (PrEC) treated with PBS, Ad-luc or Ad-mda7 were harvested 72 hours after treatment and analyzed for apoptotic cells by Annexin- $V$ staining. A significant $(P \leq .05)$ increase in the number of apoptotic cells was observed in Ad-mda7-treated tumor cells compared to PBS- and Ad-luc-treated cells. No significant increase in apoptotic cells was observed in Ad-mda7-treated PrEC cells compared to PBS- and Ad-luc-treated cells. Bars denote standard error (SE). 